\title{
Une évolution contrastée
}

L'exemple de l'enseignement supérieur dans la Fédération de Russie

Contrasting progress within higher education in the Russian Federation

Una evolución contrastada. El ejemplo de la enseñanza superior en la Federación Rusa

Arielle Bianquis

\section{(2) OpenEdition} Journals

Édition électronique

URL : https://journals.openedition.org/ries/2423

DOI : $10.4000 /$ ries. 2423

ISSN : 2261-4265

Éditeur

France Education international

Édition imprimée

Date de publication : 1 décembre 2000

Pagination : 79-89

ISSN : $1254-4590$

Référence électronique

Arielle Bianquis, "Une évolution contrastée », Revue internationale d'éducation de Sèvres [En ligne], 28 |

2000, mis en ligne le 01 décembre 2003, consulté le 09 juillet 2021. URL : http://

journals.openedition.org/ries/2423; DOI : https://doi.org/10.4000/ries.2423 


\title{
Une évolution contrastée
}

\section{L'exemple de l'enseignement supérieur dans la Fédération de Russie}

\author{
Arielle Bianquis
}

\begin{abstract}
Résumé
L'effondrement du régime politique du début des années quatre-vingt-dix s'est traduitdans l'enseignement supérieur comme dans tout le système éducatif - par l'émergence de procédures drastiques de décentralisation des moyens de gestion et la création d'établissements privés introduisant de vives concurrences. Les programmes universitaires se sont diversifiés, en se centrant de plus en plus sur les nouvelles demandes de l'économie de marché, aux dépens de l'ancienne recherche fondamentale planifiée. Un nouvel élitisme apparaît, face à l'égalitarisme prôné par l'ancien régime. Dans ce contexte de transition, le personnel éducatif est déstabilisé, ayant perdu pouvoir d'achat et prestige.
\end{abstract}

Contrasting progress within higher education in the Russian Federation

Since the collapse of the Soviet regime in the early 90 s, drastic efforts have been deployed to decentralise management structures, both within higher education as well as throughout the education system as a whole. At the same time, a growing number of private institutions have been set up, thus intensifying competition. University courses have been diversified and refocused to meet the market economy's demands, often to the detriment of Russia's traditional emphasis on centrally planned fundamental research. Elitism appears to be gradually replacing the principles of egalitarianism that prevailed under Soviet rule. At the same time, this transition - as well as the loss of their former prestige and purchasing power-has demoralised teaching staff.

Una evolución contrastada. El ejemplo de la enseñanza superior en la Federación Rusa La caída del régimen político a principios de los años noventa se tradujo - en la enseñanza superior como en todo el sistema educativo - por la emergencia de procedimientos drásticos de descentralización de los medios de gestión y la creación de centros escolares privados rivales que aumentaron la competición. Los programas universitarios se han diversificado, enfocan cada vez más las nuevas necesidades de la economía de mercado, a costa de la antigua investigación fundamental planificada. Un nuevo elitismo ha aparecido frente al igualitarismo preconizado por el antiguo régimen. En este entorno de transición, el personal educativo está desestabilizado, ya que ha perdido el poder adquisitivo y el prestigio. 
Dans une période de transition politique, la réforme de l'enseignement supérieur est une tâche essentielle afin de permettre au nouveau régime de disposer de nouvelles bases stables. Dès la perestroïka, une volonté de démocratisation des établissements universitaires était perceptible au sein du Comité d'État de l'enseignement supérieur. La loi-cadre sur l'éducation du 10 juillet 1992 constitue le texte fondateur ; il sera suivi en août 1996 d'une loi sur l'enseignement supérieur et l'enseignement professionnel post-universitaire. Ce texte autorisait pour la première fois la création d'établissements privés, il définissait également la répartition des compétences en matière d'éducation entre les différents niveaux de pouvoir (la Fédération, les régions et les municipalités) et il accordait, enfin, une réelle autonomie de gestion aux établissements existants. Ceux-ci pouvaient créer des filières payantes, constituer des structures commerciales, éducatives ou non, rentabiliser leurs locaux en les louant partiellement à des entreprises privées. Il leur était donc permis de recourir à des financements privés pour améliorer leur fonctionnement. De même une plus grande liberté dans le choix des programmes et l'organisation des cursus leur était octroyée.

Tout ceci creusait une brèche importante dans le centralisme rigide de l'administration soviétique de l'éducation dans laquelle se sont engouffrées, pour le meilleur et pour le pire, les universités russes. Les règles du système éducatif ont été profondément transformées par les réformes. L'introduction de la concurrence, la décentralisation des moyens de gestion et de décision, le désengagement de l'État, les bouleversements socio-économiques, le développement des nouvelles technologies ont amené les enseignants et les administrateurs à innover tant pour le fonctionnement quotidien des universités que pour la rénovation des cursus. En 1996, selon les chiffres de l'OCDE, on dénombrait 535 établissements publics de l'enseignement supérieur et 200 établissements privés dont 141 reconnus officiellement.

L'analyse de la situation actuelle de l'enseignement supérieur en Russie ${ }^{1}$ met en lumière des problèmes très proches de ceux de l'enseignement primaire et secondaire. Les variations se limitent souvent à une question d'échelle. Au-delà du monde éducatif, ces contradictions se retrouvent dans l'ensemble chaotique de la société russe d'aujourd'hui.

Trois axes principaux de réflexion peuvent être dégagés pour analyser les enjeux auxquels se trouve confronté l'enseignement supérieur : la rénovation des cursus a constitué le premier pas essentiel des réformes, mais il s'est accompagné d'inégalités et d'une évolution disparate des conditions de vie et de travail des enseignants.

1 Cet article s'appuie sur une centaine d'entretiens semi-directifs réalisés en 1998 et 1999 auprès d'enseignants et de responsables de l'enseignement du supérieur à Moscou, Saint-Pétersbourg et Nijni-Novgorod et sur une expérience de plus de cinq ans d'enseignement et de coopération au sein de ces deux dernières villes. 


\section{La mise en place de nouveaux programmes}

Le système soviétique était caractérisé par la planification qui évidemment se retrouvait dans l'institution universitaire. L'État définissait les priorités économiques et donc ouvrait aux concours d'entrée ${ }^{2}$ le nombre de places correspondant à ses besoins. Tout étudiant titulaire d'un diplôme de fin d'études se voyait affecté automatiquement à un emploi dans un lieu donné pendant trois ans. Libre à lui ensuite de chercher un autre poste mais la première affectation ne pouvait se refuser. Ce lien étroit entre économie planifiée et enseignement était renforcé par le rattachement statutaire d'un grand nombre d'établissements à des ministères de branche (industrie, agriculture, affaires étrangères... ), voire directement à des grandes entreprises. Ces établissements formaient à des emplois très pointus correspondant à la commande d'État. Les formations étaient très cloisonnées, aucune passerelle n'était prévue, ce qui a freiné l'adaptation aux nouvelles conditions du marché de l'emploi. La tendance actuelle, dans les pays de l'OCDE, est d' "apprendre à apprendre ", c'est-à-dire d'éviter une spécialisation étroite et de préférer une formation plus généraliste facilitant l'adaptation dans un monde où le travail, évolutif, nécessite une mise à jour régulière des connaissances ${ }^{3}$.

Les nouveaux besoins de l'économie russe nécessitaient de tout autres réponses et l'adaptation de la formation à cette évolution de la demande a été rendue encore plus difficile car elle se doublait d'une situation financière catastrophique. Les universités se sont trouvées dans l'obligation de réorganiser complètement leur fonctionnement au moment même où l'État cessait de leur fournir les moyens de subsistance les plus élémentaires. Elles ont dû chercher d'autres sources de financement comme la loi les y autorisait. Comme elles étaient généralement bien dotées en locaux de grande capacité et facilement accessibles en transports en commun, les présidents d'université et leurs adjoints ont essayé de les rentabiliser en les louant en partie aux entreprises privées en plein essor au début des années quatre-vingt-dix. Celles-ci n'avaient, le plus souvent aucun rapport avec la spécialité de l'établissement (salon de coiffure, dentiste, banque, société en informatique) et, parfois, le personnel et les étudiants montaient eux-mêmes une structure commerciale en son sein.

2 L'entrée à l'université (et dans les autres établissements d'enseignement supérieur comme les instituts ou les académies) se faisait et se fait toujours par concours. Un certain nombre de places sont offertes dans chaque discipline : en fonction des notes obtenues dans le cycle secondaire et des résultats aux épreuves, les étudiants sont admis dans telle ou telle spécialité.

3 Voir à ce sujet les recommandations de l'OCDE dans une étude réalisée entre octobre 1997 et juin 1998, publiée en 1999, Examens des politiques nationales d'éducation, enseignement tertiaire et recherche en Fédération de Russie. 
Malheureusement cette commercialisation des structures éducatives s'est faite parfois au détriment des étudiants et des conditions de travail. Ainsi des étages entiers de résidences universitaires ont été fermés pour être loués à des firmes privées, réduisant ainsi notablement les possibilités d'hébergement peu onéreuses pour ceux qui n'habitaient pas sur place.

Cette liberté, cette marge de manœuvre toute neuve pour les responsables universitaires avait des aspects stimulants car, pour la première fois, ils pouvaient prendre des initiatives et tenter de régler leurs problèmes directement sans en référer à la lourde bureaucratie ministérielle. Pourtant ce désengagement de l'État suscite un débat assez intense tant auprès des responsables éducatifs que dans les médias en général. N'est-ce pas la porte ouverte aux excès, à la corruption, aux abus de biens sociaux? Comment le personnel souvent âgé, entièrement formé sous l'ancien régime, peut-il comprendre et appliquer de façon efficace les nouvelles règles de l'économie de marché? Quelle est l'instance la plus apte à donner aux jeunes la formation nécessaire pour un bon développement du pays ? Dans la situation chaotique actuelle, ne vaudrait-il pas mieux un meilleur encadrement réglementaire pour assurer la cohésion de cette fonction fondamentale de l'éducation?

Le ministère de l'Enseignement général et professionnel, résultant de la fusion du comité d'État de l'enseignement supérieur et du ministère de l'Enseignement en 1996, a toujours été tiraillé entre une logique interventionniste et les restrictions budgétaires draconiennes auxquelles il doit faire face en cette période de crise. Il est difficile pour l'autorité de tutelle d'imposer son avis aux établissements alors qu'elle ne remplit pas ses obligations essentielles, notamment dans le domaine financier (irrégularité des versements, voire interruption totale des paiements tant pour les salaires que pour les frais de fonctionnement).

Le ministère a néanmoins mis en place un système général de certification des établissements et des formations. Tout établissement d'enseignement doit se faire enregistrer auprès de l'État qui lui accorde une licence, définissant les types de formations agréées et les diplômes qu'il est autorisé à délivrer ainsi que le nombre maximum d'étudiants qu'il peut accueillir. Cette homologation est très importante étant donné la multiplication des établissements privés en tout genre dont les prestations pédagogiques sont parfois d'un niveau douteux. La question des diplômes est centrale. Deux nouveaux cursus, l'un de quatre ans (bakalavr), l'autre de deux ans supplémentaires (magistratur) sont venus se superposer au diplôme traditionnel en cinq ans ${ }^{4}$ mais ne rencontrent guère de

4 Le fractionnement du diplôme n'étant pas possible (pas de diplôme intermédiaire avant celui de fin d'études supérieures) et le système d'unités de valeurs capitalisables n'existant pas, les étudiants doivent réussir un cycle complet de cinq ans dans leurs discipline et établissement d'origine s'ils veulent obtenir un diplôme. 
succès auprès des entreprises qui leur préfèrent l'ancien modèle plus familier. L'influence occidentale très forte dans la refonte des cursus pose de réels problèmes d'homologation et de validation des diplômes par les autorités académiques russes. Les étudiants sont souvent obligés de suivre un cursus traditionnel en parallèle pour obtenir un diplôme russe sans lequel leurs acquis ne seront jamais reconnus. Des commissions de spécialistes par discipline ont fixé les connaissances minimales devant être acquises par les étudiants au cours de chaque année d'études et dans chaque matière; il s'agit plutôt de normes en matière de connaissances et de volume horaire que d'exigences dans le domaine de l'apprentissage et des compétences. Le volume horaire est passé en 1996 de 36 à 28 heures hebdomadaires, ce qui demeure encore trop important par rapport à la nécessité d'un temps de travail personnel. Le mode d'évaluation russe reste essentiellement fondé sur l'oral (les examens écrits de réflexion sont presque inexistants), sur la mémoire, sur la rapidité de réponse sans insister suffisamment sur la réflexion personnelle, l'autonomie de l'étudiant dans l'apprentissage, l'initiation à la recherche. La forte influence du mode d'évaluation sur le mode d'apprentissage des connaissances a été démontrée maintes fois ${ }^{5}$.

Ce problème concerne d'autant plus l'enseignement secondaire que se développe depuis quelques années un système de coopération voire d'intégration entre des universités et des écoles secondaires spécialisées. Ces dernières constituent les viviers dans lesquels s'approvisionnent les établissements du supérieur, viviers qu'ils contribuent à former en envoyant certains de leurs enseignants y donner des cours. Cette intervention des universités pose également le problème d'une orientation précoce des enfants. La question préoccupe certains spécialistes : est-il réellement profitable pour un enfant de se spécialiser dès son plus jeune âge vers la banque ou le droit alors que la tendance européenne est de retarder le plus tard possible les choix définitifs de métier en prolongeant considérablement l'enseignement pluridisciplinaire. Cette spécialisation se fait en fonction des demandes du marché à court terme alors que sont ignorés les besoins du pays en vue d'un développement durable.

Les institutions privées qui ont fleuri depuis le début des années quatre-vingt-dix participent activement à ce phénomène d'engouement, de mode pour certaines formations mais elles ont également facilité l'introduction d'un enseignement novateur hors du carcan traditionnel. Plus riches que leurs homologues publics, elles ont su s'équiper rapidement afin d'utiliser les nouvelles technologies, se former aux nouvelles méthodes d'apprentissage, s’adjoindre les services des plus talentueux pédagogues obligeant ainsi les autres

5 Un exemple anecdotique, mais parlant, peut-être donné par la mise en place de centres d'examen pour l'obtention du DALF (Diplôme approfondi de la langue française) dans un certain nombre d'établissements enseignant la langue française et par les conséquences que cela a entrainées sur les modes d'apprentissage de cette matière au sein des établissements concernés avec lintroduction de nouveaux types d'exercices. 
établissements à se moderniser pour ne pas perdre une part significative de leurs effectifs. L'apparition de structures éducatives ouvertement payantes a amené un nouveau débat, celui de l'élitisme du système éducatif.

\section{Élitisme et développement de nouvelles inégalités}

Face à l'égalitarisme prôné par l'ancien régime, l'essor actuel de pratiques élitistes dans l'accès au savoir semble revêtir un caractère légitime pour les heureux bénéficiaires. Cet égalitarisme, idéologie officielle, n’était déjà plus une réalité à la fin de l'époque soviétique. Si jusqu'aux années soixante, l'école soviétique permettait une réelle mobilité sociale, ce n'était plus vrai par la suite avec, notamment, la mise en place d'écoles spécialisées en mathématiques, physique, langues. Ces écoles recrutaient les élèves les plus doués qui étaient à peu près assurés d'entrer dans l'université de leur choix ensuite. La pratique du répétiteur s'était généralisée parmi les familles qui avaient quelques moyens et pouvaient se permettre de rémunérer un enseignant du secondaire ou du supérieur pour qu'il donne des cours particuliers ou des conseils. Ceci dans le but que l'enfant réussisse le concours d'entrée. L'accès à l'éducation était officiellement ouvert à tous, mais une sélection par l'argent s'était déjà installée discrètement.

L'insuffisance dramatique des financements publics a amplifié ce phénomène. Le passage, payant, par une année préparatoire, au sein même de l'université, pour ceux qui ont raté le concours d'entrée constitue maintenant la norme. Le sponsor est aujourd'hui une figure essentielle, parent d'élève qui fait des dons en nature (ordinateurs, téléviseurs, mobilier...) ou qui fait réparer à ses frais une partie des bâtiments. Ce mécénat, qui est une manière informelle de financer certains établissements publics, en rend plus difficile l'accès pour les enfants de ceux qui n'ont pas de telles contreparties à proposer. Ce parrainage crée aussi parfois des îlots de richesse au sein d'établissements démunis.

Outre une inégalité entre les postulants, il existe une inégalité entre les établissements et, au sein de ceux-ci, entre les filières prestigieuses et celles qui ne le sont pas. L'engouement pour les métiers du tertiaire et, plus particulièrement, pour les services risque de rendre problématique la modernisation des formations techniques, si nécessaires au redéploiement de l'industrie russe, car les présidents d'universités privilégient les filières rentables ou susceptibles de soulager leurs problèmes financiers. La loi de 1992 a autorisé un effectif de $25 \%$ d'étudiants/élèves supplémentaires payant leurs études et suivant le même cursus que les autres. Ceci a introduit des inégalités entre les filières prestigieuses qui trouvent beaucoup de candidats prêts à payer leur formation et d'autres disciplines qui ne peuvent avoir accès à cette source de financement supplémentaire. Au sein des filières les plus recherchées actuellement (droit, économie, langues étrangères), les effectifs d'étudiants payant leur formation 
dépassent largement le seuil légal pour atteindre jusqu'à $90 \%$ du nombre total. En 1997, le montant moyen des droits d'inscription à l'université était estimé à 1000 dollars.

Il faut ajouter à cela que certaines universités font payer l'accès aux ressources pédagogiques comme les bibliothèques, les salles informatiques, mais aussi aux cours de langues. Le sous-investissement notable dans les ressources pédagogiques amène une situation paradoxale : l'information des scientifiques était meilleure à l'époque de la censure soviétique qu'aujourd'hui et ceci en particulier pour les sciences exactes. Les bibliothèques spécialisées ${ }^{6}$ étaient très bien fournies afin de permettre aux chercheurs d'atteindre un niveau élevé d'expertise dans leur domaine. Aujourd'hui, les budgets ne sont plus suffisants pour renouveler les abonnements à des revues étrangères et pour l'achat régulier d'ouvrages. La plupart des ouvrages ne sont accessibles qu'à ceux qui ont les moyens de les acheter ou de les faire venir de l'étranger. La liberté de voyager, d'établir des contacts avec l'étranger et l'essor d'internet ne remplacent malheureusement pas une bonne bibliothèque universitaire.

Le système de bourses ne peut compenser les inégalités d'accès à l'éducation pour les classes défavorisées. Ces bourses, qui couvrent juste le coût de l'abonnement mensuel aux transports urbains de Moscou, ne sont pas distribuées selon des critères sociaux mais à tous ceux qui n'ont pas de trop mauvaises notes. Certains se demandent si l'instauration de droits d'inscriptions obligatoires pour tous ne permettrait pas ensuite une redistribution favorisant les plus nécessiteux.

Plus d'un tiers des étudiants ne suivent leur cursus qu'à temps partiel (cours du soir, sessions mensuelles voire semestrielles, enseignement à distance), faute de moyens le plus souvent. Le facteur géographique accentue les inégalités. En 1996, le tiers des établissements d'enseignement supérieur, c'està-dire 183 sur les 535 recensés sur l'ensemble du territoire de la Fédération, se trouvait dans les régions du Centre et du Nord-Ouest. Sur ces 183 établissements, 87 étaient localisés à Moscou et 42 à Saint-Pétersbourg. Le coût des transports est devenu prohibitif pour la plupart des jeunes résidant en province ou en zones rurales. Ils ne peuvent même pas se rendre à l'examen d'entrée. Pour remédier à ce problème, certaines universités décentralisent leurs lieux d'examen pour permettre au plus grand nombre de tenter leur chance. Malheureusement le coût de la vie dans les grandes villes constitue un obstacle important pour beaucoup, et l'on trouve de moins en moins de personnes d'origine provinciale ou rurale dans les établissements des grandes villes. De plus, l'essor des conventions d'intégration des écoles aux universités handicape les enfants qui viennent de l'extérieur. Il est beaucoup plus difficile pour un

6 L'accès à certains documents jugés sensibles était restreint, mais les spécialistes de la question et les étudiants avancés pouvaient en prendre connaissance dans les fonds spéciaux des bibliothèques. 
élève excellent, mais dont la scolarité s'est déroulée hors du réseau urbain, d'accéder à un bon établissement d'enseignement supérieur que pour un élève plus médiocre, mais sociabilisé dans cet environnement porteur. Un autre contraste existe entre les régions et les villes riches qui peuvent subventionner le système éducatif local et accorder des aides substantielles aux étudiants et celles qui se trouvent démunies. De même, une ville comme Moscou offre des possibilités incomparables de revenus d'appoint, d'activités entrepreneuriales, de coopération avec le secteur privé local ou international, avec les grandes institutions russes ou étrangères.

L'aide internationale constitue, elle aussi, un facteur de développement d'un enseignement à deux vitesses. Les secteurs ayant réussi à susciter l'intérêt des Occidentaux ont reçu des subventions très importantes. Cela leur a permis de se moderniser et de rester à la pointe de leur domaine tant dans l'enseignement que dans la recherche. Le dynamisme de ces structures contraste avec la désertion d'autres facultés en ruine, ne trouvant ni moyens, ni vocations pour leurs spécialités. Cette aide a eu de nombreux aspects positifs, en permettant de conserver l'excellence dans certains domaines, de moderniser l'enseignement, le matériel, de mettre en place une coopération scientifique internationale, d'éviter une hémorragie des cerveaux. Mais elle a aussi des détracteurs qui l'accusent de siphonner le potentiel scientifique russe, de sélectionner les meilleurs pour les inciter ensuite à s'installer à l'étranger, d'orienter la recherche russe selon les intérêts des pays ou des organismes bailleurs de fonds et surtout de privilégier la recherche appliquée à court terme au détriment d'une recherche fondamentale de longue haleine (les projets étant souvent d'une durée de trois ans). La crise de l'été $1998^{7}$, puis l'intervention de l'OTAN au Kosovo ont provoqué un repli nationaliste. La situation catastrophique du pays fut imputée à l'échec des réformes impulsées par les Occidentaux. En ce qui concerne plus spécialement l'enseignement supérieur, cela a suscité des interrogations à propos du bien-fondé de l'influence étrangère sur la rénovation du système éducatif russe. Il faut ajouter qu'il y a eu de nombreux investissements étrangers dans la première moitié de la décennie quatrevingt-dix et qu'on assiste depuis quelques années à un désengagement des Occidentaux qui laisse un arrière-goût d'amertume aux collègues russes.

Cette aide était orientée vers la recherche mais également vers la modernisation des cursus par l'introduction de nouvelles méthodes d'enseignement, par la mise en place de projets de coopération entre établissements, de programmes d'échanges d'étudiants et d'enseignants. Elle constitue une source très importante de revenus pour certains établissements et surtout pour certains enseignants.

7 L'annonce, le 17 août 1998, d'une forte dévaluation du rouble accompagnée d'une suspension des remboursements de crédits étrangers provoque la faillite de nombreuses banques et la chute du pouvoir d'achat d'une grande partie de la population. 


\section{La situation du personnel éducatif}

Profession prestigieuse, relativement privilégiée sur un plan économique et social sous l'ancien régime, l'enseignement supérieur était pourtant loin de recouvrir une catégorie sociale homogène. Il existait de très importants écarts de salaires entre les différentes catégories d'enseignants au sein d'une même institution. Au début des années quatre-vingt, un enseignant débutant ou non titulaire d'un doctorat commençait avec 105 roubles, un maître de conférence avec 300 et un professeur, docteur d'État, avec 400 (ce qui constituait l'un des plus hauts salaires dans la hiérarchie soviétique). L'enseignant débutant, même titulaire d'une thèse de troisième cycle, ne pouvait vivre uniquement de son salaire: il devait le compléter avec des cours particuliers, une aide des parents, des activités diverses. Le phénomène de cumul des revenus (non déclarés pour la plupart), qui s'est généralisé depuis le début des années quatrevingt-dix, existait déjà à l'époque soviétique dans de nombreux secteurs de la société.

$\mathrm{Si}$, dans le discours communiste, l'image de l'intellectuel avait souvent une connotation négative, l'enseignant du supérieur, quant à lui, bénéficiait d'une aura spécifique qui entrainait son lot de contraintes. Le prestige du savoir se doublait d'un rôle clé dans la formation de l'homme soviétique ${ }^{8}$. Il devait donc se plier à un conformisme certain autant dans son enseignement que dans son comportement et respecter les canons en vigueur à l'époque. Aujourd'hui, s'il a gagné en liberté, il se trouve confronté à une situation socioéconomique très difficile. Les valeurs comme la situation économique ont évolué et les enseignants sont une catégorie sociale particulièrement touchée par les bouleversements de ces dernières années. La baisse très forte de niveau de vie des enseignants et la possibilité d'un accès immédiat à l'argent facile ont mis le prestige du savoir à rude épreuve. Si le salaire d'un professeur d'université titulaire atteignait le double du salaire moyen dans l'industrie en 1987, il n'en représentait déjà plus que $67 \%$ en 1993. Le cumul des emplois s'est donc imposé à tous comme une nécessité pour assurer un minimum vital à la famille. L'apparition des établissements privés a constitué une source de revenus complémentaires non négligeable pour les enseignants, leur permettant de continuer à exercer dans la même spécialité. Ce fut de même extrêmement profitable à ces nouveaux établissements qui trouvaient un personnel très qualifié sans avoir besoin de le former, ni de l'embaucher à temps plein; cela permit à ces structures de conserver une certaine souplesse qui faisait cruellement défaut aux lourdes

8 "Car l'éducation est le moyen le plus efficace dont dispose une société pour former ses membres à son image " a écrit Maurice Halbwachs dans son introduction à L'évolution pédagogique en France de E. Durkheim, PUF, Quadrige, 1999, p. 2, (1 er éd. 1938). 
bureaucraties des établissements traditionnels et à leurs effectifs pléthoriques. Ce fut surtout le cas à Moscou qui concentrait la majeure partie de ces établissements, $80 \%$ en 1996. Dans d'autres villes, les enseignants devaient trouver des compléments de salaire dans des activités ayant souvent peu de rapport avec le monde éducatif.

La qualité de l'enseignement et de la recherche s'en trouve affectée. Si l'aide internationale a permis à des enseignants de grande valeur de rester dans l'université en leur assurant des revenus décents, elle implique souvent de fréquents séjours à l'étranger qui constituent une entrave au fonctionnement dynamique et permanent d'une équipe de recherche ou d'un encadrement pédagogique. Le recours aux cours privés individuels ou collectifs tout en évitant aux enseignants de se disperser, les gêne néanmoins dans le plein exercice de leurs responsabilités au sein de leur établissement d'origine. Les professeurs sont alors, du fait de la surcharge de travail, moins disponibles pour le suivi des étudiants, la préparation des cours, la recherche. Pour les Russes, un lien substantiel existe entre la qualité de l'apprentissage et l'effectif de la classe, d'où un taux d'encadrement très important : un enseignant pour sept étudiants. Mais cela compense-t-il l'épuisement des enseignants qui ont à assurer au sein même de leur établissement de rattachement un volume horaire plus lourd que celui de leurs homologues occidentaux? Ils manquent également de temps et de ressources pour se recycler, s'informer sur les avancées dans leurs disciplines.

Ce fort taux d'encadrement implique un problème de gestion d'effectifs. Les enseignants sont en surnombre ce qui rend l'avenir de certains d'entre eux précaire. C'est particulièrement le cas du personnel le plus âgé, les retraites étant notoirement insuffisantes, le cumul d'une pension de retraite avec un service complet d'enseignement est fréquent et ce jusqu'à un âge très avancé. La population enseignante a beaucoup vieilli car si ces retraités actifs sont nombreux, la tranche 35-50 ans, quant à elle, est peu représentée au sein des établissements d'enseignement. Nombreux sont ceux qui ont quitté l'enseignement pour des emplois plus lucratifs. Ayant en général charge de famille, il leur était impossible de subvenir aux besoins de celle-ci avec les salaires modiques qu'ils touchaient à l'université. Ils gardent souvent un service minimal pour le plaisir d'enseigner, mais aussi pour conserver un emploi stable dans ce contexte incertain. Les rémunérations officielles sont à ce point symboliques, sans parler des retards dans les versements, que l'enseignement tient parfois du bénévolat. Les retraités arrivent à se satisfaire de cette double source de revenu mais les jeunes qui doivent fonder un foyer et s'équiper se trouvent dans une situation difficile. Et cela, d'autant plus, qu'il est malaisé de trouver une place pour les rares candidats désireux de s'essayer à la carrière universitaire, car personne n'a le cœur de refuser aux plus anciens de continuer leur carrière au-delà de la limite d'âge légale.

La population enseignante s'est cependant trouvée renouvelée par l'arrivée massive de chercheurs de l'Académie des sciences dont les instituts ne 
recevaient plus de subventions suffisantes pour qu'ils puissent continuer leurs travaux. L'Académie des sciences, à l'époque soviétique, était un organe où le contrôle idéologique était moins strict qu'au sein du monde universitaire car moins en prise avec le public et l'esprit y était moins conservateur. Cet apport de nouveaux spécialistes irrite souvent les universitaires traditionnels qui leur reprochent de ne pas maîtriser la pédagogie mais il n'en demeure pas moins une évolution de l'encadrement et un enrichissement de la communauté universitaire. Il est difficile de moderniser une structure quand les responsables en place sont les mêmes que sous le système précédent et qu'ils ont été entièrement formés par celui-ci, même si cela permet une continuité nécessaire.

$$
* *
$$

Durant ces dix dernières années de réformes parfois contradictoires, le système éducatif russe a cherché ses repères entre l'héritage soviétique d'un enseignement très performant mais inadapté à la société actuelle et les expériences étrangères qu'il ne paraît pas souhaitable de transposer sans les adapter. Un des exemples de tension entre différents modèles est illustré par les luttes d'influences entre les tenants d'un fort interventionnisme de l'État dans le domaine éducatif et ceux qui voudraient laisser le marché réguler l'ensemble 9 .

Malgré la baisse dramatique des financements publics, l'enseignement supérieur russe a connu une évolution remarquable ces dernières années grâce au dévouement des enseignants. Les responsables universitaires ont dû trouver localement des solutions à leurs problèmes, et ont été ainsi amenés à innover et à s'émanciper de la tutelle rigide de la bureaucratie ministérielle. Cela pose cependant un réel problème de cohérence et d'égalité tant sur un plan social que géographique, problème que les nouvelles réformes en débat actuellement viendront peut-être atténuer.

9 Voir à ce sujet l'article de Carole Sigman, dans Le Courrier des pays de l'Est du mois d'août 2000, $\mathrm{n}^{\circ} 1007$, pp. 32-47, "Quel système éducatif pour la Russie ? L'affrontement de deux modèles ». 